\title{
Long noncoding RNA-ATB promotes cell proliferation, migration and invasion in gastric cancer
}

\author{
YING CHEN $^{1 *}$, GUOQING WEI $^{2 *}$, HONGWEI XIA ${ }^{2}$, QIULIN TANG $^{2}$ and FENG BI ${ }^{1,2}$ \\ ${ }^{1}$ Department of Medical Oncology, West China Hospital, Sichuan University; ${ }^{2}$ Laboratory of Signal Transduction and \\ Molecular Targeted Therapy, State Key Laboratory of Biotherapy, Sichuan University, Chengdu, Sichuan 610041, P.R. China
}

Received December 31, 2016; Accepted August 8, 2017

DOI: $10.3892 / \mathrm{mmr} .2017 .8077$

\begin{abstract}
Long noncoding RNA (lncRNA)-activated by transforming growth factor (TGF)- $\beta$ (lncRNA-ATB) was recognized as an unfavorable prognostic factor in various cancers; however, its regulatory role in gastric cancer (GC) remains elusive. The present study aimed to measure lncRNA-ATB expression in GC and to explore its involvement in GC progression. IncRNA-ATB expression levels were measured in 40 pairs of $\mathrm{GC}$ tissues and their normal adjacent tissues, as well as in 5 GC cell lines and a normal gastric mucosal cell line by reverse transcription-quantitative polymerase chain reaction. Knockdown experiments were performed to explore the effect of IncRNA-ATB on the cell proliferation, invasion and migration. The results demonstrated that lncRNA-ATB expression levels in GC tissues and GC cell lines were significantly higher than in the adjacent normal tissues and normal gastric mucosal cells. Further analysis of the correlation between the clinicopathological features and lncRNA-ATB expression indicated that higher expression of lncRNA-ATB was correlated with increased invasion depth, more distant metastasis and advanced tumor-node-metastasis stage. In addition, downregulated lncRNA-ATB expression suppressed cellular proliferation, invasion and migration of GC cells. In conclusion, these data suggested that IncRNA-ATB may serve as a clinical outcome predictor and potential therapeutic target in GC.
\end{abstract}

\section{Introduction}

Gastric cancer (GC) is the fifth most common cancer and the third most common cause of cancer-related mortality

Correspondence to: Professor Feng Bi, Department of Medical Oncology, West China Hospital, Sichuan University, 37 Guoxue Road, Chengdu, Sichuan 610041, P.R. China

E-mail: bifeng@medmail.com.cn

${ }^{*}$ Contributed equally

Key words: long noncoding RNA, lncRNA-ATB, transforming growth factor- $\beta$, gastric cancer, epithelial-to-mesenchymal transition worldwide (1). As GC development is a multistep carcinogenic process that involves numerous genetic and epigenetic alterations, a better understanding of oncogenes and tumor suppressor genes may provide additional clues for understanding the underlying molecular mechanism for GC development and for molecular targeted drug screening (2).

Long noncoding RNAs (lncRNAs) are a class of non protein-coding transcripts that are $>200$ nucleotides in length (3). A number of studies have demonstrated the vital role of IncRNA expression dysregulation in numerous diseases, particularly in cancer, owing to its extensive biological functions in a wide variety of physiological and pathological processes $(4,5)$. IncRNA-activated by transforming growth factor (TGF)- $\beta$ (IncRNA-ATB), was identified during a screen of IncRNAs that are regulated by TGF- $\beta$ in hepatocellular carcinoma (HCC); the study indicated that the IncRNA-ATB was upregulated in HCC (6). In addition, lncRNA-ATB was demonstrated to promote epithelial-to-mesenchymal transition (EMT) and induce the invasion-metastasis cascade through the TGF- $\beta$ /microRNA (miR)-200s/zinc-finger E-box-binding homeobox (ZEB) axis, which contributes to HCC progression (6). A series of subsequent studies demonstrated that upregulated lncRNA-ATB expression was correlated with poor prognosis in several cancer types, including GC (7), renal cell carcinoma (8), colorectal cancer (9) and breast cancer (10). However, the role of lncRNA-ATB in EMT process and invasion-metastasis cascade in GC remains unknown.

The present study investigated the expression of lncRNA-ATB in GC tissues and cell lines, as well as its relationship with clinicopathological features of patients with GC, with a focus on the specific modulatory roles of lncRNA-ATB in the proliferation, invasion and migration of GC cells.

\section{Materials and methods}

Specimens and relative clinical data. A total of 40 pairs of GC tissues and adjacent non-tumor tissues, along with the patients' clinical data were obtained from the Tissue Bank in West China Hospital, Sichuan University (Chengdu, China). All tissues were stored in liquid nitrogen until used for RNA extraction. This study was approved by the Research Ethics Committee of West China Hospital, and written informed consent was received from all patients prior to enrollment in the present study. 
Cell culture. Five human GC cell lines (MGC-803, MKN-45, BGC-823, MKN-28 and SGC-7901) and a normal gastric mucosal cell line (GES-1) were acquired from our laboratory depository, originally obtained from the American Type Culture Collection (ATCC; Manassas, VA, USA). All cell lines were cultured in Dulbecco's modified Eagle's medium (DMEM; Gibco; Thermo Fisher Scientific, Inc., Waltham, MA, USA) supplemented with $10 \%$ fetal bovine serum (FBS; Gibco; Thermo Fisher Scientific, Inc.) and incubated in a humid atmosphere of $5 \% \mathrm{CO}_{2}$ at $37^{\circ} \mathrm{C}$. The medium was replaced every 2 days.

$R N A$ extraction and reverse transcription-quantitative polymerase chain reaction ( $R T-q P C R)$ analysis. The NucleoZOL Reagent (Machery-Nagel GmbH, Düren, Germany) was used to extract total RNA from the cell lines $\left(10^{7}\right.$ cells $\left./ \mathrm{ml}\right)$ and patient tissue specimens $(100 \mathrm{mg}$ tissue $/ \mathrm{ml})$. The concentration and purity of RNA were detected with a NanoDrop 2000 spectrophotometer (NanoDrop Technologies; Thermo Fisher Scientific, Inc., Wilmington, DE, USA). Reverse transcription of total RNA into cDNA was performed by PrimeScript RT Reagent kit with gDNA Eraser (Takara Bio, Inc., Otsu, Japan), following the manufacturer's instructions. qPCR was performed using 2X ChamQ SYBR qPCR Master Mix (Vazyme Biotech Co., Ltd., Nanjing, China) with a total reaction volume of $10 \mu \mathrm{l}$. qPCR was performed on a Bio-Rad CFX96 Touch Real-Time PCR Detection System (Bio-Rad Laboratories, Inc., Hercules, CA, USA). Thermocycling conditions were as follows: One cycle of $95^{\circ} \mathrm{C}$ for $30 \mathrm{sec}$, followed by 40 cycles of $95^{\circ} \mathrm{C}$ for $10 \mathrm{sec}$ and $60^{\circ} \mathrm{C}$ for $30 \mathrm{sec}$. The primer sequences used were as follows: IncRNA-ATB forward, $5^{\prime}$-TCT GGCTGAGGCTGGTTGAC-3' and reverse, 5'-ATCTCTGGG TGCTGGTGAAGG-3'; internal housekeeping gene GAPDH forward, 5'-TTGGTATCGTGGAAGGACTCA-3' and reverse, 5'-TGTCATCATATTTGGCAGGTT-3'. Relative expression levels of lncRNA-ATB were calculated by the $2^{-\Delta \Delta \mathrm{Cq}}$ method, normalized to GAPDH (11). The ratio value of each sample was subsequently determined as the ratio of expression between $\mathrm{GC}$ and normal tissue from the same patient. Each experiment was performed in triplicate.

$R N A$ interference. The lncRNA-ATB-targeted small interfering (si)RNA and a non-specific negative control (si-NC) were both purchased from Shanghai GenePharma Co., Ltd. (Shanghai, China). The sequence of the si-IncRNA-ATB was: 5'-CCAUGAGGAGUACUGCCAATT-3'. MGC-803 and MKN-45 cells were seeded in a 6 well plate $\left(0.5 \times 10^{6}\right.$ cells/well $)$ and then transfected with $100 \mathrm{nM}$ si-lncRNA-ATB or si-NC using Lipofectamine 2000 (Invitrogen; Thermo Fisher Scientific, Inc.) according to the manufacturer's instructions. Following $48 \mathrm{~h}$ incubation at $37^{\circ} \mathrm{C}$, the transfected cells were harvested for further analysis.

Cell proliferation assay. Cell proliferation was examined by Cell Counting Kit-8 (CCK-8; Dojindo Molecular Technologies, Inc., Shanghai, China), following the manufacturer's instructions. Briefly, MGC-803 and MKN-45 cells were seeded in a 96-well plate $\left(1 \times 10^{4}\right.$ cells/well) $24 \mathrm{~h}$ prior to transfection with $100 \mathrm{nM}$ si-lncRNA-ATB or si-NC using Lipofectamine 2000. Following $48 \mathrm{~h}$ of transfection at $37^{\circ} \mathrm{C}$, serum free DMEM
(100 $\mu$ l) containing 10\% CCK-8 reagent was added to each well and cultured for $1 \mathrm{~h}$ at $37^{\circ} \mathrm{C}$. The absorbance at a wavelength of $450 \mathrm{~nm}$ was assessed with a microplate reader (Bio-Rad Laboratories, Inc.). Experiments were repeated at least three times.

Ethynyl-2-deoxyuridine (EdU) incorporation assay. Cell proliferation was also examined by EdU incorporation using the Cell-Light Apollo Stain kit (Guangzhou RiboBio Co., Ltd., Guangzhou, China), according to the manufacturer's instructions. MGC-803 and MKN-45 were seeded in a 96-well plate ( $1 \times 10^{4}$ cells/well) $24 \mathrm{~h}$ prior to transfection with $100 \mathrm{nM}$ si-lncRNA-ATB or si-NC using Lipofectamine 2000. Following $48 \mathrm{~h}$ incubation at $37^{\circ} \mathrm{C}$, EdU $(100 \mu \mathrm{l} ; 50 \mu \mathrm{M})$ was added into each well and incubated for $2 \mathrm{~h}$ at $37^{\circ} \mathrm{C}$. The cells were fixed in of $4 \%$ paraformaldehyde in PBS $(100 \mu \mathrm{l})$ for $20 \mathrm{~min}$ at room temperature. Subsequently, the cells were incubated in glycine $(50 \mu \mathrm{l} ; 2 \mathrm{mg} / \mathrm{ml})$ for $5 \mathrm{~min}$, followed by washes with PBS. Cells were permeabilized with $1 \%$ Triton X-100 and incubated with 1X Apollo Solution for $45 \mathrm{~min}$ at the room temperature in dark. Hoechst solution (20 $\mu \mathrm{l})$ was added into each well and incubated for an additional $10 \mathrm{~min}$ at room temperature in dark, followed by washes with PBS. Plates were observed and images captured under a Nikon Eclipse TE2000-U fluorescence microscope (Nikon Corporation, Tokyo, Japan); magnification, x20. The excitation and emission parameters of Apollo are 550 and $565 \mathrm{~nm}$, respectively. The excitation and emission parameters of Hoechst are 350 and $461 \mathrm{~nm}$, respectively. Experiments were repeated at least three times.

Transwell invasion and migration assays. MGC-803 and MKN- 45 cells were seeded in a 6 -well plate $\left(0.5 \times 10^{6}\right.$ cells/well $)$ and transfected with $100 \mathrm{nM}$ si-lncRNA-ATB or si-NC using Lipofectamine 2000 according to the manufacturer's instructions. Following $48 \mathrm{~h}$ incubation at $37^{\circ} \mathrm{C}$, the transfected cells were resuspended in serum-free DMEM and added in the upper Transwell chamber with a Matrigel coated membrane. For the migration assay, cells were seeded into the upper chamber without Matrigel. The bottom chamber was filled with $400 \mu \mathrm{l}$ DMEM containing 20\% FBS. Cells were incubated for $48 \mathrm{~h}$ at $37^{\circ} \mathrm{C}$. Non-invading cells were wiped off of the upper surface with a wet cotton swab, and the cells that passed to the lower surface were fixed in $4 \%$ paraformaldehyde at room temperature for $20 \mathrm{~min}$ and then stained with $0.5 \%$ crystal violet solution at room temperature overnight. Images of the invaded cells were captured under an Eclipse T1-SM inverted microscope (Nikon Corporation) at x10 magnification. Experiments were repeated at least three times.

Wound-healing assay. The migratory ability of GC cells was examined by wound-healing assay. At $48 \mathrm{~h}$ post-siRNA transfection, MGC-803 and MKN-45 were seeded in a 6-well plate $\left(0.5 \times 10^{6}\right.$ cells/well $)$. The cells were allowed to reach $90 \%$ confluence, after which a scratch was made through the center of each well using the $200 \mu 1$ sterile pipette tip. The scratch was observed and imaged at 0,24 and $48 \mathrm{~h}$ following the scratch using an Eclipse T1-SM inverted microscope (Nikon Corporation) with x10 magnification. Experiments were repeated at least three times. 
Western blot analysis. Cellular proteins of transfected MGC-803 and MKN-45 cells were lysed on ice with radioimmunoprecipitation assay lysis buffer (BioTeke Corporation, Beijing, China) according to the manufacturer's instructions. The concentration of protein was detected using a NanoDrop 2000 spectrophotometer (NanoDrop Technologies; Thermo Fisher Scientific, Inc., Pittsburgh, PA, USA). Equal amounts of protein extracts (20 $\mu \mathrm{g} / \mathrm{lane})$ were separated by $8 \%$ SDS-PAGE and transferred electrophoretically to a polyvinylidene difluoride membrane (EMD Millipore, Billerica, MA, USA) using the Bio-Rad Semi-dry Transfer System (Bio-Rad Laboratories, Inc.). Membranes were blocked with 5\% milk in PBS for $2 \mathrm{~h}$ at room temperature and probed with specific primary antibodies $(1: 1,000)$ against $\beta$-actin (cat. no. ab8227), vimentin (cat. no. ab45939), N-cadherin (cat. no. ab18203) and c-Myc (cat. no. ab11917) (all from Abcam, Cambridge UK), at $4^{\circ} \mathrm{C}$ overnight. Following 3 washes with PBS (5 min each), IRDye 650-conjugated goat anti-mouse (cat. no. 926-68070)/rabbit (cat. no. 926-32210) immunoglobulin G secondary antibodies (1:200; LI-COR Biosciences, Lincoln, NE, USA) were added and incubated for $1 \mathrm{~h}$ in the dark at room temperature. Membranes were washed with PBS and bands were detected in a dark room using a Fluorescence Odyssey Imaging System (LI-COR Biosciences).

Statistical analysis. Analysis of the correlation between patient clinicopathological characteristics and lncRNA-ATB expression was performed using SPSS 22.0 software (IBM Corp., Armonk, NY, USA). The remaining data analysis was performed by GraphPad Prism 5.0 (GraphPad Software, Inc., La Jolla, CA, USA). Comparison between two groups was performed using a Student's t-test. Chi-square test was used for analyzing the correlation between lncRNA-ATB expression and clinicopathological features of the GC samples. Data was presented as the mean \pm standard deviation. All of the P-values were two-tailed, and $\mathrm{P}<0.05$ was considered to indicate a statistically significant difference.

\section{Results}

IncRNA-ATB is overexpressed in GC tissues and cell lines. The expression levels of lncRNA-ATB in 40 pairs of gastric tissues were measured by RT-qPCR. A total of 40 ratios value were obtained from 40 pairs of tissues, and the average value as 4.37 . The 40 pairs were divided into a high-expression group ( $\mathrm{n}=20$; ratio $>4.37$ ) and a low-expression group $(\mathrm{n}=20$; ratio $<4.37$ ) according to the average ratio value (average ratio=4.37; Fig. 1A). IncRNA-ATB expression was significantly higher in GC tumor tissues compared with expression in the adjacent non-tumor tissues $(\mathrm{P}<0.001$; Fig. 1B). In addition, IncRNA-ATB expression was significantly higher in GC tissues from patients with distant metastasis ( $\mathrm{P}<0.01$; Fig. $1 \mathrm{C})$, whereas 1 ncRNA-ATB expression was not significantly correlated with lymph node metastasis $(\mathrm{P}>0.05$; Fig. 1D). The expression of lncRNA-ATB was also examined in different GC cell lines and the GES-1 normal gastric cell line. The results demonstrated that lncRNA-ATB expression was higher in the GC cell lines compared with expression in GES-1 (Fig. 1E).
Table I. Correlation between lncRNA-ATB expression level and clinicopathological features in patients with gastric cancer.

\begin{tabular}{lrrrr}
\hline & \multicolumn{4}{c}{$\begin{array}{c}\text { LncRNA-ATB } \\
\text { expression }\end{array}$} \\
\cline { 3 - 4 } $\begin{array}{l}\text { Clinical } \\
\text { characteristics }\end{array}$ & $\mathrm{n}$ & Low & High & P-value \\
\hline Age (years) & & & & 0.751 \\
$>60$ & 18 & 10 & 8 & \\
$<60$ & 22 & 10 & 12 & \\
Sex & & & & 0.514 \\
Male & 25 & 14 & 11 & \\
Female & 15 & 6 & 9 & \\
Invasion depth & & & & $\mathbf{0 . 0 0 3}$ \\
T1 & 4 & 3 & 1 & \\
T2 & 11 & 8 & 3 & \\
T3 & 12 & 8 & 4 & \\
T4 & 13 & 1 & 12 & \\
Lymph node & & & & 0.070 \\
metastasis & & & & \\
0 & 16 & 12 & 4 & \\
1 & 13 & 4 & 9 & \\
2 & 7 & 3 & 4 & \\
3 & 4 & 1 & 3 & \\
Metastasis & & & & $\mathbf{0 . 0 0 8}$ \\
Yes & 7 & 0 & 7 & \\
No & 33 & 20 & 13 & $\mathbf{0 . 0 0 1}$ \\
TNM stage & & & & \\
I & 13 & 11 & 2 & \\
II & 12 & 7 & 5 & \\
III & 8 & 2 & 6 & \\
IV & 7 & 0 & 7 & \\
& & & & \\
\hline
\end{tabular}

lncRNA-ATB, long noncoding RNA-activated by transforming growth factor- $\beta$; TNM, tumor-node-metastasis.

Correlation between lncRNA-ATB expression and clinicopathological characteristics in patients with GC. High IncRNA-ATB expression levels were positively correlated with invasion depth $(\mathrm{P}=0.003)$, distant metastasis $(\mathrm{P}=0.008)$ and tumor-node-metastasis (TNM) stage ( $\mathrm{P}=0.001)$, compared with the low-expression group (Table I). However, no significant correlation was identified between the IncRNA-ATB expression and lymph node metastasis, distant metastasis, patient age and sex $(\mathrm{P}>0.05)$. Therefore, it was concluded that the overexpression of IncRNA-ATB may be associated with GC progression.

IncRNA-ATB-knockdown suppresses cellular proliferation. RT-qPCR results indicated that the level of IncRNA-ATB expression was significantly reduced in GC cells transfected with si-IncRNA-ATB compared with cells transfected with si-NC ( $\mathrm{P}<0.01$; Fig. 2A), which indicated that si-lncRNA-ATB could be used in further in vitro experiments. CCK-8 assay results demonstrated that cellular proliferation was significantly 


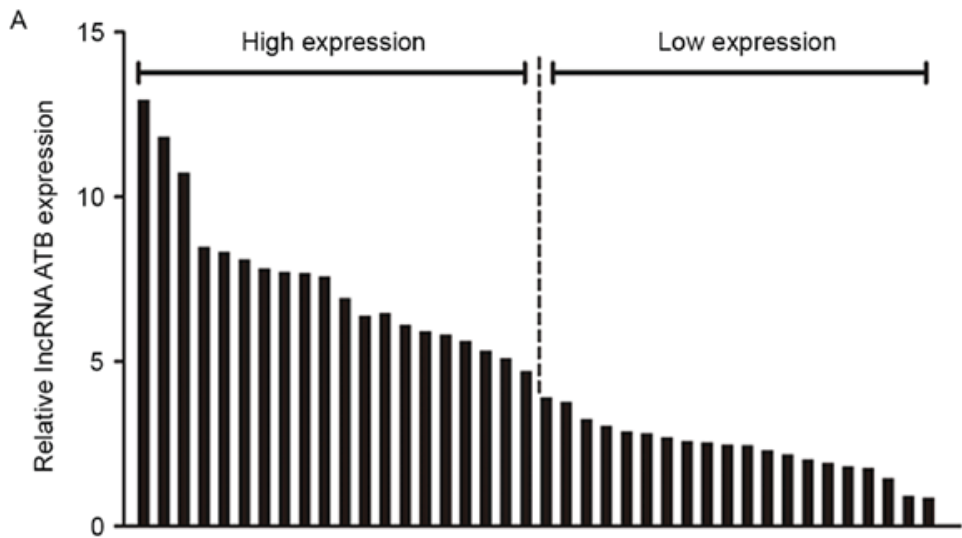

Gastric cancer tissue number ( 1 to 40 )
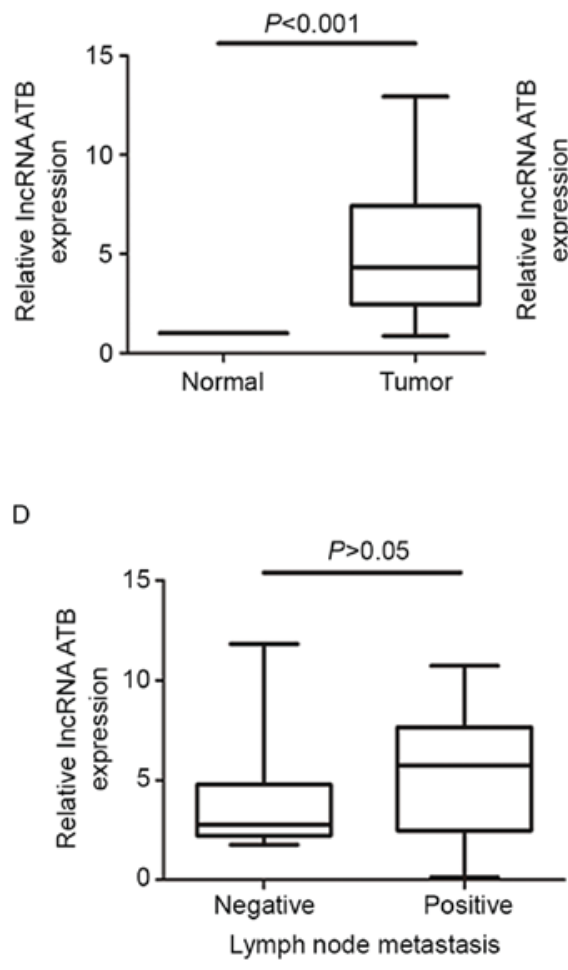

C
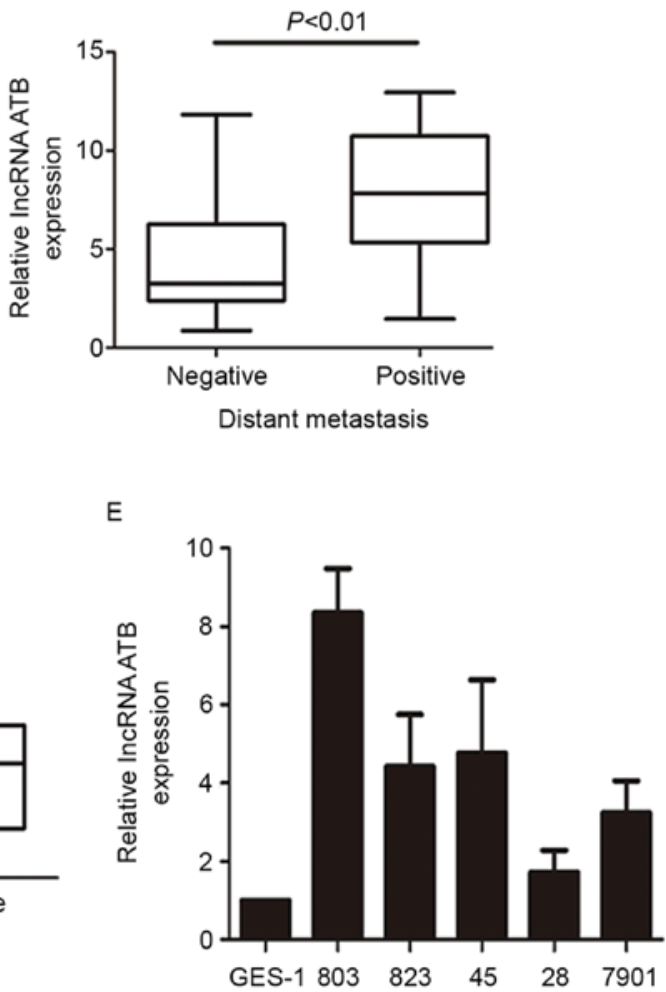

Figure 1. IncRNA-ATB is overexpressed in GC tissues and cell lines. (A) The relative expression of each GC tissue was normalized to the paired normal tissue; two groups (high and low expression) were determined according to the average value. (B) Relative expression of lncRNA-ATB in GC tissues and adjacent normal tissues. (C) IncRNA-ATB expression between localized (negative) and metastatic (positive) GC patients. (D) lncRNA-ATB expression between GC patients with (positive) and without (negative) lymph node metastasis. (E) Relative expression levels of lncRNA-ATB in GC cell lines (MGC-803, BGC-823, MKN-45, MKN-28 and SGC-7901) and normal gastric mucosal cell line (GES-1). Data are presented as the mean \pm standard deviation. All data are representative of three independent experiments. GC, gastric cancer; lncRNA-ATB, long noncoding RNA-activated by transforming growth factor- $\beta$.

suppressed in MGC-803 and MKN-45 cells transfected with si-lncRNA-ATB compared with si-NC transfected cells ( $\mathrm{P}<0.01$; Fig. 2B). The EdU assay also demonstrated reduced proliferative ability in MGC-803 cells (Fig. 2C) and in MKN-45 cells (Fig. 2D) transfected with si-lncRNA-ATB. Western blotting revealed that the expression of c-Myc, an indicator of cell proliferation, was notably reduced in the si-lncRNA-ATB group compared with the si-NC group (Fig. 2E).

IncRNA-ATB-knockdown reduces the invasion and migration ability of GC cells. In the wound healing assays, the migratory ability of both the MGC-803 cells (Fig. 3A) and the
MKN-45 (Fig. 3B) cells transfected with si-lncRNA-ATB was notably reduced compared with the control si-NC transfected cells. To further examine the effects on cell migration and invasion, Transwell assays were performed. Knockdown of lncRNA-ATB resulted in fewer cells migrating through the chambers in both MGC-803 and MKN-45 cells (Fig. 3C). Similar results were obtained with Matrigel invasion assays (Fig. 3D). As IncRNA-ATB is a TGF- $\beta$-induced lncRNA, the effects of lncRNA-ATB on the EMT process were analyzed. It was demonstrated that the silencing of lncRNA-ATB resulted in the downregulated protein expression of the mesenchymal markers N-cadherin and vimentin (Fig. 3E). 
A

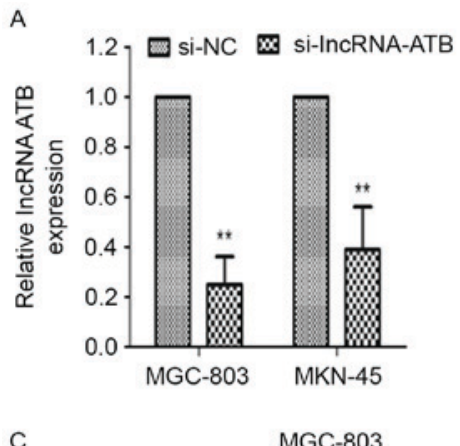

C
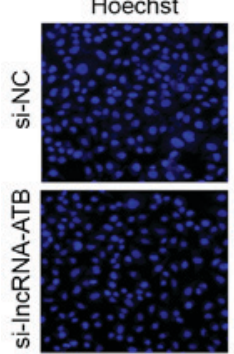

MGC-803
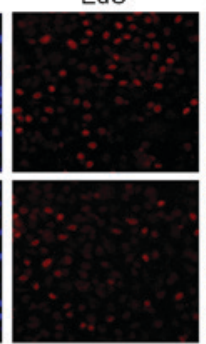

E

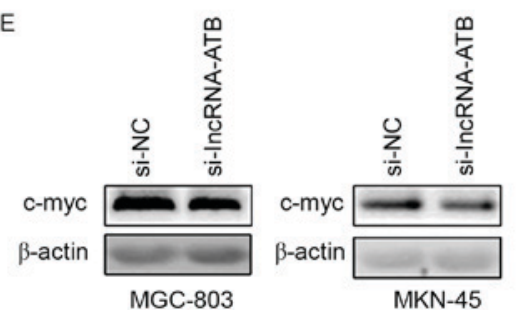

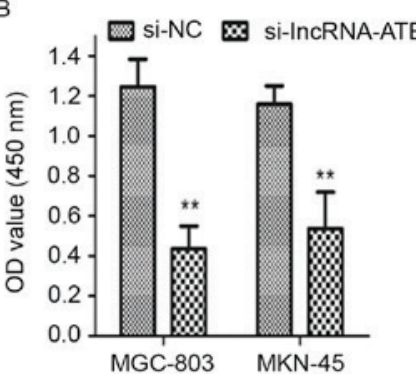

D
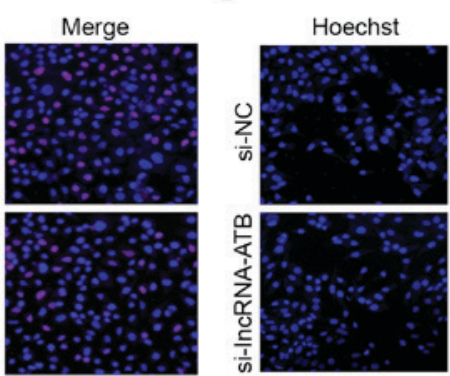

MKN-45

EdU
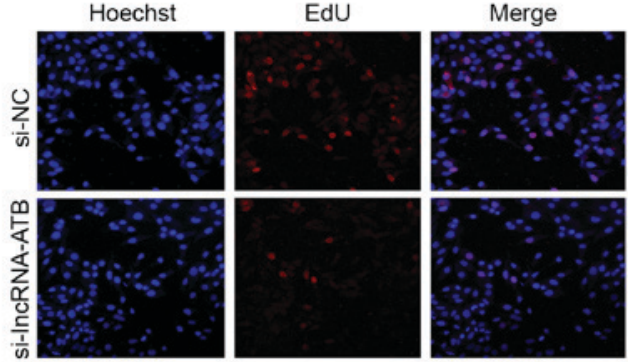

Figure 2. IncRNA-ATB-knockdown suppresses cellular proliferation. (A) Relative lncRNA-ATB expression of lncRNA-ATB in MGC803 and MKN-45 gastric cancer cells transfected with si-lncRNA-ATB compared with si-NC transfected cells. (B) Cell Counting Kit-8 was used to examine cell viability at $48 \mathrm{~h}$ post-transfection in both MGC-803 and MKN-45 cells. (C and D) EdU incorporation assay was performed to further examine cell proliferation at 48 h post-transfection in (C) MGC-803 and (D) MKN-45 cells; red-labeled cells indicate the cells undergoing the proliferation process; magnification, x20 (E) Western blot analysis for the expression of c-Myc in MGC-803 and MKN-45 cells. Data are presented as the mean \pm standard deviation; data are representative of three independent experiments. ${ }^{* *} \mathrm{P}<0.01$. lncRNA-ATB, long noncoding RNA-activated by transforming growth factor- $\beta$; NC, negative control; OD, optical density; siRNA, small interfering RNA.
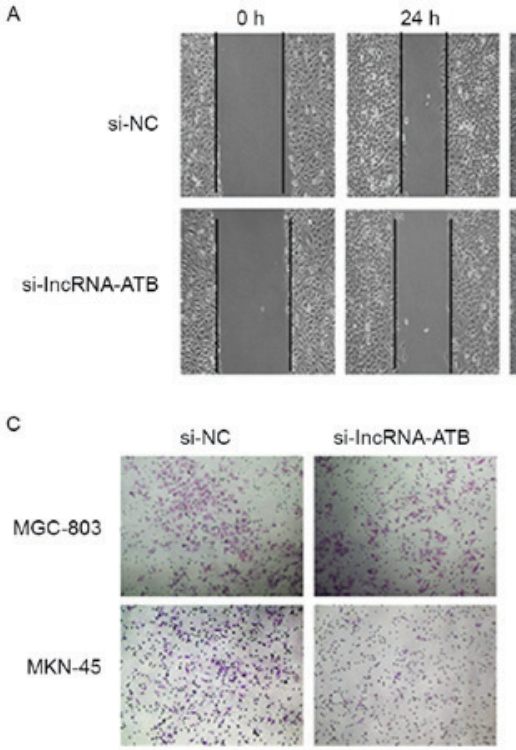

$24 \mathrm{~h}$
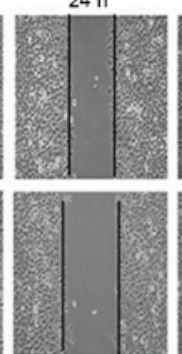

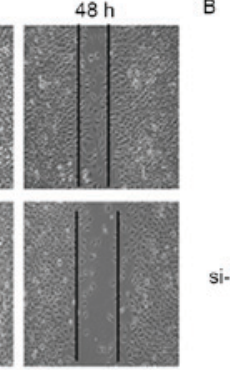

B

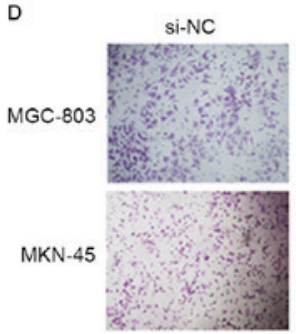

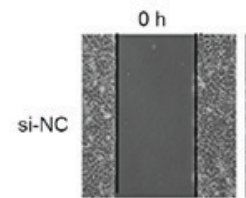

si-IncRNA-ATB

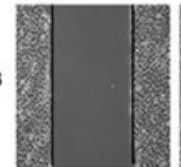

si-IncRNA-ATB

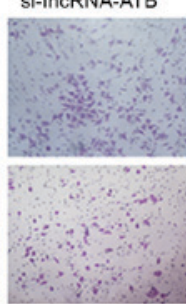

$24 \mathrm{~h}$

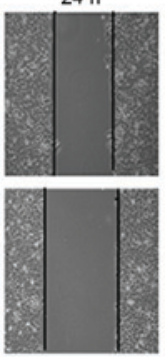

$48 \mathrm{~h}$
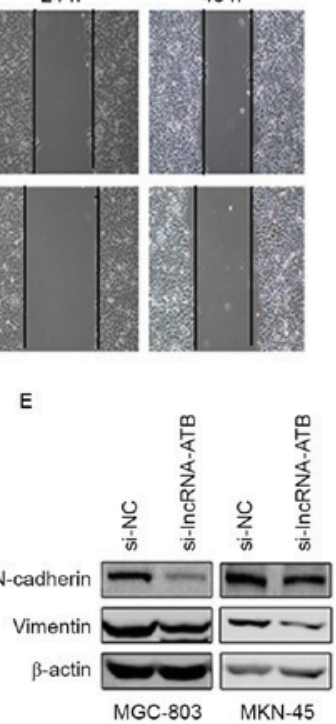

Figure 3. IncRNA-ATB-knockdown reduces the invasion and migration ability of GC cells. (A and B) Wound healing assays demonstrated the reduced migration ability in in (A) MGC-803 and (B) MKN-45 cells treated with si-lncRNA-ATB compared with si-NC control treated cells. (C) Cell migration was measured by Transwell assays with normal chambers; representative images were presented. (D) Cell invasion ability was measured by Transwell assays with Matrigel-coated chambers; representative images were presented. (C and D) Magnification, x10. (E) Western blot analysis demonstrated reduced protein expression levels of N-cadherin and vimentin in si-lncRNA-ATB-treated cells compared with si-NC control treated cells. lncRNA-ATB, long noncoding RNA-activated by transforming growth factor- $\beta$; NC, negative control; siRNA, small interfering RNA. 


\section{Discussion}

The clinical outcome of patients with GC remains dismal with the 5-year survival rate as low as $25 \%$, or less, despite the rapid progress in developing surgical techniques and molecular-targeted therapies (12). This may be attributed to the underlying highly complex molecular mechanisms, which involve multiple tumor suppressors and oncogenes, as well as some well-studied tumor-related signaling pathways (13). Therefore, it is essential to identify reliable molecular biomarkers of GC to improve the early diagnosis and overall survival rate. In addition, it may also provide additional clues to the development of individualized therapy.

Noncoding RNA was previously regarded as transcriptional 'noise' in the conventional opinion of gene regulation (14); however, an increasing number of studies have demonstrated their regulatory potential in a range of physiological and pathological processes (15). IncRNA-ATB was originally reported as a novel TGF- $\beta$-induced lncRNA that was highly overexpressed in HCC. IncRNA-ATB competitively binds to members of the miR-200 miRNA family, which results in the upregulated expression of ZEB1 and ZEB2 mRNA and protein, thus inducing EMT (6). Notably, knock down lncRNA-ATB expression was revealed to sufficiently abolish TGF- $\beta$-induced EMT in HCC cells, even with the involvement of many other TGF- $\beta$-induced EMT drivers, such as Snail, Twist and Slug $(6,16)$. These results indicated an essential role of lncRNA-ATB in EMT regulation. Upregulated lncRNA-ATB expression levels were also demonstrated in GC (7) renal cell carcinoma (8), colorectal cancer (9) as well as breast cancer (10) tissues compared with the levels of expression in the paired normal tissues. Survival analysis also indicated the inverse relationship between lncRNA-ATB expression and cancer prognosis (17). However, lncRNA-ATB expression was reported to be decreased in pancreatic cancer tissues compared with the adjacent normal tissues, which suggested the role of IncRNA-ATB in tumor progression may be pro-tumorigenic or tumor suppressive (18). Interestingly, the similar duality of TGF- $\beta$ signaling was also observed in the pancreatic ductal adenocarcinoma (19).

In the present study, lncRNA-ATB expression levels in GC tissues and GC cell lines were significantly higher compared with adjacent normal tissues and normal gastric mucosal cells. These results were consistent with a previous study (7). In addition, patients with distant metastasis exhibited high expression of IncRNA-ATB. Further correlational analysis with the clinicopathological features indicated that higher lncRNA-ATB expression was correlated with increased invasion depth, more distant metastasis and advanced TNM stage. However, a previous study reported no significant association between high lncRNA-ATB expression and depth of tumor invasion, distant metastasis or clinical stage (7). This contradiction may be due to differences in the high and low expression ratio groupings; analysis with a larger sample size is necessary to further investigate this.

The present study also performed in vitro experiments to investigate the role of lncRNA-ATB in GC cellular processes. As the MGC-803 and MKN-45 cell lines exhibited the highest expression of IncRNA-ATB, they were selected for in vitro experiments. The results indicated that the knockdown of IncRNA-ATB expression significantly suppressed the proliferation, migration and invasion abilities of GC cell lines. In addition, reduced expression levels of mesenchymal markers $\mathrm{N}$-cadherin and vimentin were observed in cells with knocked-down lncRNA-ATB expression. These data suggested that the regulatory role of lncRNA-ATB in GC progression was fulfilled partially through the induction of EMT. However, the underlying regulatory mechanism requires further clarification.

In conclusion, the present study reported that the higher expression of IncRNA-ATB were positively correlated with invasion depth, distant metastasis and advanced TNM stage. In addition, it was demonstrated that knocking down lncRNA-ATB expression reduced the proliferation, migration and invasion ability of GC cell lines. These results suggested the potential role of IncRNA-ATB in clinical outcome prediction and molecular-targeted therapy in GC.

\section{Acknowledgements}

We would like to thank the contributions of all authors who participated in this study. This study was supported by the National Natural Science Foundation of China (grant no. 81572731).

\section{References}

1. Ferlay J, Soerjomataram I, Dikshit R, Eser S, Mathers C, Rebelo M, Parkin DM, Forman D and Bray F: Cancer incidence and mortality worldwide: Sources, methods and major patterns in GLOBOCAN. Int J Cancer 136: E359-E386, 2015.

2. Wu WK, Cho CH, Lee CW, Fan D, Wu K, Yu J and Sung JJ: Dysregulation of cellular signaling in gastric cancer. Cancer Lett 295: 144-153, 2010

3. Rönnau CG, Verhaegh GW, Luna-Velez MV and Schalken JA: Noncoding RNAs as novel biomarkers in prostate cancer. Biomed Res Int 2014: 591703, 2014.

4. Guttman M and Rinn JL: Modular regulatory principles of large non-coding RNAs. Nature 482: 339-346, 2012.

5. Brunner AL, Beck AH, Edris B, Sweeney RT, Zhu SX, Li R, Montgomery K, Varma S, Gilks T, Guo X, et al: Transcriptional profiling of long non-coding RNAs and novel transcribed regions across a diverse panel of archived human cancers. Genome Biol 13: R75, 2012.

6. Yuan JH, Yang F, Wang F, Ma JZ, Guo YJ, Tao QF, Liu F, Pan W, Wang TT, Zhou CC, et al: A long noncoding RNA activated by TGF- $\beta$ promotes the invasion-metastasis cascade in hepatocellular carcinoma. Cancer Cell 25: 666-681, 2014.

7. Saito T, Kurashige J, Nambara S, Komatsu H, Hirata H, Ueda M, Sakimura S, Uchi R, Takano Y, Shinden Y, et al: A long non-coding RNA activated by transforming growth factor- $\beta$ is an independent prognostic marker of gastric cancer. Ann Sug Oncol 3 (Suppl 22): S915-S922, 2015.

8. Xiong J, Liu Y, Jiang L, Zeng Y and Tang W: High expression of long non-coding RNA lncRNA-ATB is correlated with metastases and promotes cell migration and invasion in renal cell carcinoma. Jpn J Clin Oncol 46: 378-384, 2016.

9. Iguchi T, Uchi R, Nambara S, Saito T, Komatsu H, Hirata H, Ueda M, Sakimura S, Takano Y, Kurashige J, et al: A long noncoding RNA, IncRNA-ATB, is involved in the progression and prognosis of colorectal cancer. Anticancer Res 35: 1385-1388, 2015.

10. Shi SJ, Wang LJ, Yu B, Li YH, Jin Y and Bai XZ: LncRNA-ATB promotes trastuzumab resistance and invasion-metastasis cascade in breast cancer. Oncotarget 6: 11652-11663, 2015.

11. Livak KJ and Schmittgen TD: Analysis of relative gene expression data using real-time quantitative PCR and the 2(-Delta Delta C(T)) method. Methods 25: 402-408, 2001.

12. Saka M, Morita S, Fukagawa T and Katai H: Present and future status of gastric cancer surgery. Jpn J Clin Oncol 41: 307-313, 2011. 
13. Kang W, Cheng AS, Yu J and To KF: Emerging role of Hippo pathway in gastric and other gastrointestinal cancers. World J Gastroenterol 22: 1279-1288, 2016.

14. Fang XY, Pan HF, Leng RX and Ye DQ: Long noncoding RNAs: Novel insights into gastric cancer. Cancer Lett 356: 357-366, 2015.

15. Mattick JS: RNA regulation: A new genetics? Nat Rev Genet 5: 316-323, 2004

16. Burk U, Schubert J, Wellner U, Schmalhofer O, Vincan E, Spaderna $S$ and Brabletz T: A reciprocal repression between ZEB1 and members of the miR-200 family promotes EMT and invasion in cancer cells. EMBO Rep 9: 582-589, 2008.
17. Yue B, Qiu S, Zhao S, Liu C, Zhang D, Yu F, Peng Z and Yan D: LncRNA ATB mediated E-cadherin repression promotes the progression of colon cancer and predicts poor prognosis. J Gastroenterol Hepatol 31: 595-603, 2016.

18. Qu S, Yang X, Song W, Sun W, Li X, Wang J, Zhong Y, Shang R, Ruan B, Zhang Z, et al: Downregulation of lncRNA-ATB correlates with clinical progression and unfavorable prognosis in pancreatic cancer. Tumour Biol 37: 3933-3938, 2016.

19. David CJ, Huang YH, Chen M, Su J, Zou Y, Bardeesy N, Iacobuzio-Donahue CA and Massagué J: TGF- $\beta$ tumor suppression through a lethal EMT. Cell 164: 1015-1030, 2016. 